\title{
La investigación en el tratamiento psicológico de las adicciones: en busca de la utilidad clínica
}

\author{
Emilio Sánchez Hervás \\ Unidad de Conductas Adictivas de Catarroja \\ Agencia Valenciana de Salud. Conselleria de Sanidad \\ Enviar correspondencia a: \\ Emilio Sánchez Hervás. Unidad de Conductas Adictivas - Centro de Salud. Avd. Rambleta, 63. CP 46470. Catarroja. Valencia \\ E-mail: esanchez455k@cv.gva.es
}

Recibido: 12 de septiembre de 2005. Aceptado: 6 de febrero de 2006.

\section{RESUMEN}

Tradicionalmente ha habido un distanciamiento importante entre la investigación y la práctica clínica, y se hace necesario que la investigación clínica ponga un mayor énfasis en la efectividad y la utilidad clínica de los tratamientos en adicciones. Los desafíos actuales para fomentar un uso más extendido de los tratamientos empíricamente validados, giran en torno a la generalización, la aplicabilidad y la rentabilidad. En un esfuerzo por facilitar aspectos como la utilidad y el sentido práctico, algunos autores proponen modelos mixtos de investigación que incluyan los componentes de los estudios de eficacia (rigurosidad metodológica), y los de la efectividad (investigación en contextos clínicos reales).

Poder disponer de la evidencia científica de las opciones psicoterapéuticas existentes, ayuda a emplear rutinariamente las alternativas que produzcan los mejores resultados clínicos, lo que eleva la calidad asistencial prestada a los pacientes. La integración de los acercamientos de la eficacia y la efectividad a través de programas de investigación clínicos, es necesaria para alcanzar la mejor evidencia científica.

Palabras clave: adicciones, eficacia, efectividad, tratamientos

\section{ABSTRACT}

Traditionally there has been a significant distance between research and clinical practice, and it is becoming increasingly necessary for clinical investigation to place greater emphasis on the effectiveness and the clinical utility of addiction treatments. The present challenges to promote a more extensive use of empirically validated treatments revolve around generalisation, applicability and effectiveness. In an effort to facilitate such aspects as utility and practicality, some authors propose mixed investigation models that include the components of the efficacy studies (methodological rigour), and those of the effectiveness (investigation in real clinical contexts).

The availability of the scientific evidence for the existing psychotherapeutic options aids in routinely using the alternatives that produce the best clinical results, which raises the quality of the attention given to patients. The integration of the approaches to efficacy and effectiveness, through clinical research programmes, is necessary to obtain the best scientific evidence.

Key words: addictions, efficacy, effectiveness, treatments

\section{INTRODUCCIÓN}

$\mathbf{L}$ a gestión y la dotación de recursos humanos y económicos para la mejora de la calidad asistencial es responsabilidad de políticos y gestores, y la aplicación de las técnicas y los programas de intervención es (en parte), responsabilidad de los clínicos. Durante mucho tiempo los clínicos (de diversas disciplinas) han realizado su trabajo basándose fundamentalmente en lo recomendado en los libros de texto clásicos, en la opinión de las autoridades científicas de su entorno, en su sentido común y en su experiencia personal. Esta situación ha generado que durante años las decisiones hayan sido tomadas en muchas ocasiones según el principio de la beneficencia, al no disponer de sólidas bases científicas para muchas de las intervenciones terapéuticas disponibles. Con el objetivo de paliar esta situación, a principios de la década de los noventa surgió un movimiento entre clínicos y epidemiólogos que se denominó medicina basada en la evidencia, y que ha sido adoptada por otras disciplinas (en nuestro caso Psicología Basada en la Evidencia ó también Psicología Basada en Pruebas-PBP) (Pascual, Frías y Monterde 2004), cuya filosofía se basa en la 
integración y la conjunción de la experiencia clínica personal, con la mejor evidencia científica disponible (obtenida a través de estudios de investigación clínica publicados en la literatura científica), tras una revisión exhaustiva y crítica de la misma (Rosenberg y Donald, 1995). De esta manera, las decisiones tomadas en la práctica clínica diaria estarían basadas en la mejor evidencia disponible, siendo una garantía de una buena práctica clínica. Una cuestión que no queda clara y es motivo de controversia, es qué evidencia debería ser considerada como la más idónea y relevante: la que proporcionan los ensayos clínicos controlados (eficacia), o si también deberían ser tenidas en cuenta otras evidencias, como la que nos suministran los estudios naturalísticos (efectividad), los estudios de evaluación económica (eficiencia), los estudios cualitativos (preferencias de los pacientes, calidad de vida, etc.), los estudios que valoran la indicación adecuada y el uso apropiado de las alternativas existentes (estudios de idoneidad), y los estudios que tratan de evaluar las implicaciones sociales, legales y éticas derivadas del uso de las diferentes intervenciones y/o tecnologías (Soto, 1999).

Por otra parte, en los tiempos actuales en los que es clara la limitación progresiva de los recursos disponibles que pueden ser empleados en la atención sanitaria, junto a un crecimiento exponencial e ilimitado de las necesidades, es preciso considerar dos aspectos relacionados con cualquier opción terapéutica: su eficiencia (comparando costes con resultados), y los aspectos subjetivos que describen la manera en que los pacientes perciben su efecto sobre la salud: grado de preferencia y satisfacción, calidad de vida producida, comodidad de administración y grado de accesibilidad (Pope y Mays, 1995). Respecto de la eficiencia quizás en un futuro no muy lejano sea necesario analizar como los costes se relacionan con los resultados del tratamiento. Si un programa de tratamiento ahorra más que consume, será más fácil defenderlo cómo forma de inversión social que pueda merecer más atención y fondos adicionales (NIDA, 1999). El tratamiento del abuso y la adicción a drogas es una intervención rentable comparada a otras intervenciones en el ámbito de la salud, aunque los estudios de rentabilidad no son muy frecuentes y muestran resultados dispares. En general, con mucha probabilidad los programas ambulatorios y los más cortos son los más rentables (Machado, 2005). En especial se recomiendan los programas libres de droga en contexto ambulatorio, al ser la modalidad menos costosa y no haber diferencias en la eficacia del tratamiento respecto a otras modalidades de intervención (Mojtabai y Zivin, 2003).

\section{LIMITACIONES DE LOS ESTUDIOS DE EFICACIA Y EFECTIVIDAD}

El uso sistemático de la Psicología Basada en Pruebas -PBP- permite reforzar el principio de autonomía, ya que el paciente podrá tomar una decisión basada en la mejor evidencia disponible sobre las diferentes alternativas disponibles; llevar a la práctica el principio de la no maleficencia, al poder ofertar al paciente las alternativas que producen los mejores beneficios terapéuticos, y promover el principio de justicia al garantizarse un correcto uso de los recursos disponibles para la atención sanitaria. El poder disponer de la evidencia científica de las opciones terapéuticas existentes, nos ayudará a emplear rutinariamente las alternativas que produzcan los mejores resultados clínicos, lo que indudablemente elevará la calidad asistencial prestada a los pacientes, fin último de cualquier sistema de salud (Soto, 1999). El gran reto de la PBP es llegar a incorporar estas nuevas evidencias dentro de sus principios y prácticas.

Cabe entonces plantearse algunas preguntas. ¿Es el tratamiento psicológico para el abuso y la adicción a drogas eficaz?. ¿Son todos los programas de tratamiento igualmente eficaces?. ¿Porqué los programas se diferencian en su eficacia?. ¿Qué tratamientos son los más rentables?. El tratamiento es eficaz, pero no todos los tratamientos son eficaces. Las investigaciones sobre la eficacia han versado desde el estudio de las características de los pacientes y los programas, hasta la búsqueda de los ingredientes activos del tratamiento (filosofia del tratamiento, actitudes de los profesionales hacia los pacientes, etc).

Muchos de los tratamientos para los cuales no se dispone de evidencia en la literatura científica se utilizan con frecuencia en la clínica. La demostración de la eficacia de las intervenciones psicológicas es uno de los desafíos más relevantes de la psicología clínica. Una tentativa significativa para establecer una base de evidencia científica fue llevada a cabo por la división 12 de la APA (Chambles, Sanderson, Shoham, BennetJhonson, Pope, Cris-Cristoph, et al. 1996; Chambless y Hodson, 1998; APA, 2000). Dichos trabajos dieron lugar al desarrollo de criterios para las investigaciones controladas de evaluación de los tratamientos en psicoterapia, y una lista de los tratamientos específicos para desórdenes específicos, en los cuales se habían hallado suficientes datos sobre su eficacia. Entre las críticas a este acercamiento estaba el reconocimiento de que los ensayos controlados no poseían la validez externa adecuada, por lo que los resultados no serían generalizables a otras poblaciones, serían inaplicables y no servirían como base para la toma de decisiones clínicas y políticas. Algunas de las limitaciones en el ámbito de las conductas adictivas incluyen la alta 
comorbilidad, la heterogeneidad diagnóstica, las dificultades en la adhesión al tratamiento y, la elevada presión asistencial (Sánchez-Hervás, 2001; 2004).

Tradicionalmente ha habido un distanciamiento importante entre la investigación y la práctica clínica, y se hace necesario que la investigación clínica ponga un mayor énfasis en la investigación de la efectividad y la utilidad clínica de los tratamientos (Beutler y Howard, 1998; Norquist, G. Lebowitz, B. Hyman, S., 1999; Wells, 1999), puesto que la disparidad entre investigación y práctica son particularmente evidentes en el tratamiento de las adicciones. El ejemplo reciente más claro ha sido el del tratamiento con metadona, que a pesar de contar con una abrumadora evidencia empírica sobre su eficacia y efectividad, han tenido que transcurrir varios años para que se trate de una alternativa de tratamiento sin restricciones. De igual forma algunos tratamientos psicológicos con apoyo empírico han sido poco utilizados, mientras que otros que se utilizan con cierta frecuencia no han experimentado ninguna evolución en la evaluación de su eficacia.

Los desafíos para fomentar un uso más extendido de los tratamientos empíricamente validados giran en torno a varios factores: la generalización (a profesionales, pacientes y contextos), aplicabilidad (tipo de entrenamiento y a qué profesionales), rentabilidad (costes en comparación a otros tratamientos). Los estudios de eficacia se critican con frecuencia por su limitada generabilidad a los problemas del mundo real, mientras que los estudios de efectividad resaltan las características ecológicas del diseño, pero tiene dificultades para eliminar explicaciones alternativas a los resultados.

Establecer un efecto causal requiere de los ensayos aleatorizados al azar, controlados y de doble ciego. Sin embargo la validez interna que ofrecen estos estudios es sólo la mitad de lo que se necesita. La aplicación en la práctica clínica real requiere de medidas de validez externa, demostrando que las ventajas se pueden generalizar a una población real. Además otras variables extrañas afectan a los resultados del tratamiento (exactitud del diagnóstico, aceptabilidad y accesibilidad al tratamiento). Aunque los estudios de efectividad se muestran como la prueba verdadera de la generabilidad, ésta es una asunción discutible (Klein y Smith, 1999). Varios obstáculos limitan la capacidad de los diseños naturalisticos para determinar si los tratamientos son realmente eficaces, incluso si están precedidos por estudios de eficacia la validez externa es cuestionable. La generabilidad depende de una serie de estudios que demuestren efectividad, con evaluaciones continuas de los resultados. Los estudios no pueden reflejar la realidad clínica si la evaluación es un adjunto a la práctica, más que una parte integral del tratamiento. La carga de trabajo no permi- te incorporar fácilmente responsabilidades adicionales de evaluación. El control de calidad es extremadamente dífícil.

Aunque los métodos y las estrategias para la evaluación de la eficacia con ensayos controlados se ha definido bien en la investigación en los tratamientos psicológicos y farmacológicos (Rounsaville, B. CarroII, K. Onken, L., 2001; Insitute of Medicine, 2001), no está tan clara cuales deben de ser las estrategias para la investigación de la efectividad. Además, el impacto de los resultados de los estudios de efectividad sobre la comunidad clínica y los pagadores (agencias, políticos) ha sido escaso. Esta carencia de impacto puede ser debido a varios factores, como la falta de preguntas críticas sobre la utilidad de los tratamientos en la práctica clínica o los defectos metodológicos que afectan a la validez interna.

Se ha propuesto el uso de resultados clínicos en la práctica clínica habitual, como estrategia que facilite la valoración de las medidas de cambio que se producen en los pacientes a lo largo del tratamiento. El manejo de resultados clínicos se refiere a la práctica que hace uso de medidas estandarizadas para evaluar sistemáticamente los cambios en el estado clínico de los pacientes a lo largo del tiempo. En general, las medidas de resultados usados en la investigación académica tienden a requerir demasiado tiempo de administración, y son prohibitivamente caras para su uso en contextos prácticos. Las medidas de resultados usadas en los centros sanitarios y por los profesionales de la salud deben de ser fáciles de calcular, tener un costo bajo por administración, alta sensibilidad para los cambios en el malestar psicológico en períodos de tiempo breves, y limitarse a características claves asociadas con el funcionamiento de la salud mental. La respuesta a esta necesidad de medir resultados clínicos válidos y prácticos en centros sanitarios debe generar el desarrollo de medidas que se adapten mejor a las necesidades del profesional que busca la efectividad frente a los resultados ofrecidos por los estudios de eficacia (Sammons, 2004).

\section{ESTUDIOS COMBINADOS DE EFICACIA Y EFECTIVIDAD}

Los ensayos controlados de eficacia están siendo cuestionados duramente, y se plantea que tienen que ser abandonados porque no tienen una adecuada validez externa. Los estudios de efectividad realizados en clínicas de tratamiento con pacientes reales se defienden como diseño alternativo. Se plantea la adopción de un modelo de clínicas en el que se estudie la eficacia y la efectividad de los tratamientos psicológicos en adicciones. En un esfuerzo por facilitar mayor énfasis en aspectos como la utilidad y el sentido práctico, 
algunos autores han propuesto modelos híbridos de investigación para fomentar un uso más amplio de los tratamientos empíricamente validados en los programas de tratamiento del abuso de sustancias, a la vez que realzar la producción científica de la investigación sobre la eficacia de los tratamientos (Carroll y Rounsaville, 2003). El modelo híbrido conserva las características esenciales de la investigación de la eficacia, mientras que amplia las preguntas de la investigación para tratar el estudio de la efectividad.

Los diseños híbridos propuestos por algunos autores incluyen los elementos de los ensayos de eficacia, conservando el rigor científico a través de preservar las características del diseño que protejan la validez interna (asignación al azar de los pacientes al tratamiento, uso de medidas objetivas de resultados, definición y supervisión de los tratamientos utilizados, entrenamiento especializado de los terapeutas). Aunque estas características aumentan el coste y la complejidad de los ensayos clínicos las desventajas de omitirlos son numerosas y relevantes. La más importante de ellas es que los estudios que no asignan aleatoriamente los participantes a una condición de tratamiento pierden la capacidad de eliminar las explicaciones alternativas a los resultados (pérdida de validez interna), por lo que la generalización posterior es discutible (Carroll y Rounsaville, 2003). Así, tendrían que conservarse los componentes de la eficacia, agregándose los componentes de la efectividad (diversidad de pacientes y contextos de tratamiento, rentabilidad del tratamiento, satisfacción de los pacientes y de los clínicos), usando criterios menos restrictivos de inclusión o exclusión.

Para el desarrollo de investigaciones de ensayos clínicos y de efectividad es necesario redefinir tanto los objetivos de la investigación como los métodos, incluyendo los análisis económicos de coste-eficacia. El desarrollo de diseños de tipo mixto que combinen las características de eficacia y efectividad requerirá la identificación de diversos aspectos como la frecuencia del seguimiento, la rigurosidad de las mediciones y el tamaño muestral (Wells, 1999).

Aunque la difusión de tratamientos se ha estudiado en otros campos, no se aplica a menudo en el campo de la adicción a drogas. Los resultados de la investigación de la difusión de innovaciones puede ayudar a mejorar las lagunas existentes en la investigación (Gotham, 2004; Fals-Steward, W. Logson, T. Birchler, G., 2004). Diversas son las complejidades en la difusión de los tratamientos empíricamente validados a los contextos "verdaderos de tratamiento". Una cuestión importante es saber qué clase de entrenamiento deben de tener los clínicos para aplicar con eficacia los tratamientos validados, por lo que también se requieren estudios empíricos de los métodos de entrenamiento. Otro aspecto importante es el de dilucidar los costes del entrenamiento y del tratamiento de la adicción en sí. El valor de los análisis de la rentabilidad es sugerido por varios estudios que han demostrado el ahorro y las ventajas asociadas a un tratamiento (Svikis, D. Golden, A. Guggins, G., 1997; Avants, S. Margolin, A. Sindelar, J., 1999; Holder, Cisler y Longabaugh, 2000). Cuando la disminución de los costes se demuestra, los pagadores están más interesados en la adopción de esos tratamientos. Se sugiere que en los análisis económicos de los tratamientos de la adicción se consideren los denominados "resultados múltiples", porque centrarse en un solo resultado, puede conducir a inferencias inadecuadas y posiblemente incorrectas.

Un último aspecto a destacar es el énfasis reciente sobre la preferencia y la satisfacción de los pacientes como indicadores de la utilidad y el valor de un tratamiento (McLellan y Hunkeler, 1998; Holcomb et al, 1998; Rohland, Langbehn y Rohrer, 2000). Los indicadores de la satisfacción de los pacientes son importantes en la determinación de si un nuevo acercamiento agregaría valor a un programa haciéndolo más atractivo a los pacientes. La mayor intensidad y satisfacción con el servicio ha sido relacionada positivamente con la terminación del tratamiento, o una retención más larga en éste con resultados favorables. Esto se dá tanto en pacientes en programas libres de droga, en pacientes no internados y en pacientes residenciales. Mayor intensidad del servicio y satisfacción de los pacientes, terminación del tratamiento y retención, son elementos del proceso que mejoran los resultados del tratamiento (Hser, Evans, Huang y Anglin, 2004).

\section{DISCUSIÓNY CONCLUSIONES}

Los pacientes y los pagadores están especialmente interesados en los efectos de los tratamientos en la práctica clínica, al margen de los resultados de los ensayos clínicos rigurosos. Para alcanzar un conocimiento adecuado de ello se necesita una amplia agenda de investigación que incluya estudios de eficacia, estudios de efectividad y estudios híbridos que incluyan características de ambos. La aparición de está nueva perspectiva refleja un cambio subyacente del paradigma hacia una mayor preocupación de los impactos sociales del tratamiento y hacia un reexámen de lo que se considera evidencia científica relevante en los tratamientos. Ello supone la creación de programas de investigación clínicos que maximizarían la validez interna de las investigaciones al utilizar metodologías rigurosas, y que proporcionarían validez externa al realizar las investigaciones en contextos clínicos reales. Algunas experiencias ya se han realizado en este sentido (Borkovec, 2001).

Las soluciones potenciales a los problemas relacionados con la utilidad clínica se relacionan con la identificación de las terapias aplicadas que sean 
equivalentes a los tratamientos eficaces, evaluando la efectividad de dichas terapias o estandarizándolas para aproximarlas a los tratamientos eficaces. Este es un desafío importante en la investigación sobre efectividad, estandarizar intervenciones preservando los cuidados necesarios a los pacientes, el ajuste clínico y el contexto de la intervención; sin embargo no existen estándares para ello. Un estándar mínimo podría incluir una descripción del nivel de formación de los terapeutas, disponibilidad de los recursos y presencia o ausencia de estructuras de gestión (sistemas de calidad) (Wells, 1999). Es necesario documentar sistemáticamente el contexto del tratamiento y procurar identificar factores del contexto que puedan afectar a los resultados del tratamiento. La organización del tratamiento afecta a los resultados finales.

Las tendencias actuales en la investigación sobre los tratamientos en las adicciones reflejan las preocupaciones en la obtención de la evidencia científica más generalizable de los efectos del tratamiento, en aplicar tratamientos eficaces en contexto reales de intervención, y en los diseños de evaluación de los tratamientos que enfatizan los resultados (funcionamiento, coste-utilidad) (Berglund, Andreasson, Fmack, Fridell, Hakanson et al. 2001). Estas tendencias están conduciendo a un mayor énfasis de estudios aplicados con menor protección de la validez interna y mayor focalización en la validez externa, aunque la generalización es dificil de alcanzar. Ello requerirá estudios más grandes y costosos. La integración de los acercamientos de la eficacia y la efectividad en estudios híbridos es necesaria.

Por lo que respecta a nuestra realidad más cercana, las dificultades en la investigación de los tratamientos psicológicos en adicciones se relacionan además de lo ya comentado anteriormente con otras "variables" adicionales que apuntamos a continuación. Por un lado podría mencionarse el escaso interés de las distintas administraciones y de los gestores por todo lo relacionado con "lo psicológico". No olvidemos el conflicto actual a propósito de la consideración de la Psicología como profesión sanitaria, y la lentitud y diferencia de criterios que se están utilizando en la recalificación como facultativos especialistas de los psicólogos clínicos en la administración sanitaria. Si a ello le añadimos la escasa importancia que desde las Facultades de Psicología parece atribuírsele a la labor de los psicólogos en el tratamiento de las adicciones (ver el estudio de Lloret, Poveda y Solivares, (2004) sobre la docencia de las drogodependencias en la universidad española), no es difícil de adivinar en que estado se encuentra la investigación en el área: escasa, limitada a ámbitos universitarios y por tanto, poco aplicada.

Una posible solución a estas dificultades sería que los propios clínicos se decidieran a investigar por sí solos, pero ni existe una formación adecuada para ello, ni se disponen de las infraestructuras necesarias adecuadas. Ello, sin mencionar la conocida presión asistencial en los recursos sobre drogodependencias, y el tiempo limitado que se dispone para la labor clínica, no digamos ya para la investigación.

Cabe añadir que los resultados de la investigación actuales parecen indicar que los programas de tratamiento más efectivos son los que combinan terapia conductual y psicofármacos (NIDA, 2004), por lo que los tratamientos combinados se presentan como un área prioritaria en la investigación en adicciones. Los clínicos tendremos que mejorar nuestra formación en investigación, y hacer un esfuerzo adicional en nuestra labor clínica para poder dedicar tiempo a tareas a las que tradicionalmente no hemos prestado atención. Pero ello no será suficiente sin que las instituciones (administración, universidad, colegios de psicólogos, sociedades científicas, etc) cuenten con los clínicos como investigadores, participando en la definición de las áreas prioritarias de investigación, en la elaboración de proyectos y en la toma de decisiones.

\section{REFERENCIAS}

APA. American Psychological Association. (2000). Society of Clinical Psychology. A Guide to Beneficial Psychotherapy. Empirically Supported Treatments. Washington: American Psychological Association.

Avants, S. Margolin, A. Sindelar, J. (1999). Day treatment versus enhanced standard methadone services for opioid-dependent patients: a comparison of clinical efficacy and cost. American Journal of Psychiatry 156:27-33.

Berglund, M. Andreasson, S. Frnack, J. Fridell, M. Hakanson, I. et al. (2001). Treatment of alcohol and drug abuse. And evidence based review. Swedish Council on Technology Assesment in Health Care. Report 156/1, $156 / 2$.

Beutler, L. Howard, K. (1998). Clinical utility research: an introduction. Journal of Clinical Psychology 54:297-301.

Borkovec,T. Echemendia, R. Ragusea, S. Ruiz, M. (2001). The Pensylvania practice research network and future posibilities clinically meaningful and scientifically rigorous psychotherapy effectiveness research. Clinical Psychology: Sciencie and Practice, vol 8(2): 35-39

Carroll, K. Rousanville, B. (2003). Bridgin the gap: a hibryd model to link efficacy and effectiveness research in substance abuse treatment. Psychiatric Services, 54: 333-339.

Chambless, D. Hodson, S. (1998). Defining empirically supported therapies. J. of Consulting and Clinical Psychology, 66: 3-18.

Chambless, D. Sanderson, W. Shoham, V. Bennet-Jhonson, S. Pope, K. Cris-Cristoph, P. Baker, M. Jhonson, B. Woody, S. Sue, S. Beutler, L. Williams, D. McCurry, 
S. (1996). A update on empirically validated therapies. Clinical Psychologist, 49: 5-22.

Coulter, A. Jenkinson, C. (2005). European patients' views on the responsiveness of health systems and healthcare providers. Eur J Public Health, 23.

Disney, H. (2004). Impatient for change: european attitudes to healthcare reform. Stochkolm Network

Fals-Steward, W. Logson, T. Birchler, G. (2004). Difusion of and empirically supported treatment for substance abuse: an organizational autopsy of technology transfer sucess and failure. Clinical Psychology, 11(2): 190-195.

Gotham, H. (2004). Difusion of mental health and substance abuse treatments: development, dissemination, and implementation. Clinical Psychology, 11(2): 160-176.

Holcomb, W. Parker, J. Leong, G. (1998). Customer satisfaction and self-reported treatment outcomes among psychiatric inpatients. Psychiatric Services 49:1622-1623.

Holder, H. Cisler, R. Longabaugh, R. (2000) Alcoholism treatment and medical care costs for Project MATCH. Addiction 95:999-1013.

Hser, Y. Evans, E. Huang, D. Anglin, D. (2004). Relationship between drug treatment services, retention and outcomes. Psychiatric Services, 5: 767-774

Institute of Medicine. (2001). Small Clinical Trials: Issues and Challenges. Washington, DC, National Academy Press.

Klein, D. Smith, L. (1999). Organizational requirements for effective clinical effectiveness studies. Prevention and Treatment, 2: 50-55.

Lloret, D. Poveda, M. Solivares, M. (2004). Formación pregrado en drogodependencias en las universidades españolas. Adicciones, vol 16, n³: 197-206.

Machado, M. (2005). Substance abuse treatment, what do we know?. An economist prespective. Eur. J. Health Econ, 6(1): 53-64

McLellan, A. Hunkeler, E. (1998). Patient satisfaction and outcomes in alcohol and drug abuse treatment. Psychiatric Services 49:573-575.

Mojtabai, R. Zivin, J. (2003). Effectiveness and costeffectiveness or four treatment modalities for substance disorders: a propensity score analysis. Health Serv Res, 38(1): 233-259.

NIDA. (1999). Measuring and improving costs, costeffectiveness, and cost-benefit for substance abuse treatment programs. National Institute on Drug Abuse. National Institute of Health.
NIDA. (2004). Developing effective addiction treatments. Nida Notes, vol 9 (1).

Norquist, G. Lebowitz, B. Hyman, S. (1999). Expanding the frontier of treatment research. Prevention and Treatment, 2: 44-48

Pascual, J. Frías, M. Monterde, H. (2004). Tratamientos psicológicos con apoyo empírico y práctica clínica basada en la evidencia. Papeles del Psicólogo, 87: 1-8

Pope, C. Mays, N. (1995). Reaching the parts other methods cannot reach: an introduction to qualitative methods in health and health services research. Br Med J, 311: 42-45

Rohland, B. Langbehn, D. Rohrer, J. (2000). Relationship between service effectiveness and satisfaction among persons receiving Medicaid mental health services. Psychiatric Services 51:248-250.

Rosenberg, W. Donald, A. (1995). Evidence-based medicine: an approach to clinical problem-solving. Br Med J; 310 : 1.122-1.126

Rounsaville, B. Carroll, K. Onken, L. (2001). A stage model of behavioral therapies research: getting started and moving on from stage I. Clinical Psychology: Science and Practice 8:133-142.

Sammons, M. (2004). Tratamientos combinados en los trastornos mentales: dilemas clínicos. En: Tratamientos combinados de los trastornos mentales: una guía de intervenciones psicológicas y farmacológicas. Sammons, M. y Schmidt, N. (eds). Bilbao. DDB.

Sánchez-Hervás, E. (2001). Tratamientos psicológicos basados en la evidencia y conductas adictivas. Boletín de Psicología, 71: 63-77

Sánchez-Hervás, E. (2004). Tratamientos psicológicos en adicciones: eficacia, limitaciones y propuestas para mejorar su implantación. Papeles del Psicólogo, n 87: 34-44.

Soto, J. (1999). Medicina basada en la eficiencia: una inevitable realidad para el siglo XXI. Medicina Clinica, 113: 655-657.

Svikis, D. Golden, A. Guggins, G. (1997). Cost-effectiveness of treatment for drug-abusing pregnant women. Drug and Alcohol Dependence 45:105-113.

Wells, K. (1999). Treatment research at the crossroads: the scientific interface of clinical trials and effectiveness research. American Journal of Psychiatry 156:5-10.

Zeithaml, V. Parasuraman, A. Berry, L. (1993). Calidad total en la gestión de servicios. Madrid: Díaz de Santos. 